\title{
A Brainstem Network Mediating Apneic Reflexes in the Rat
}

\author{
Nancy L. Chamberlin and Clifford B. Saper \\ Department of Neurology, Harvard Medical School and Beth Israel Hospital, Boston, Massachusetts 02115
}

Apnea is an important protective response to upper airway irritation, but the central mechanisms responsible for eliciting sensory-induced apnea are not well understood. Recent studies have emphasized the Kölliker-Fuse nucleus in producing apnea and proposed a trigeminoparabrachial pathway for mediating these reflexes. However, in our earlier study of apneic responses produced by glutamate stimulation in the dorsolateral pons, we found that apnea was elicited from the area just ventral to the Kölliker-Fuse nucleus, rather than within it. Because this region was not known to be involved in respiratory control, we combined chemical microstimulation with both anterograde and retrograde axonal tracing to characterize the sites in the pons that produce apneic responses. We found that apneic sites were consistently associated with the intertrigeminal region, between the principal sensory and motor trigeminal nuclei. Injections of anterograde tracer at these sites labeled terminals in the ventral respiratory group, in the ventrolateral medulla. Injection of retrograde tracer into this target region in the ventrolateral medulla disclosed a previously unrecognized population of neurons among the trigeminal motor rootlets. Injection of retrograde tracer into this intertrigeminal region demonstrated inputs from portions of the spinal trigeminal nucleus and the nucleus of the solitary tract that have been associated with producing sensory apnea. Our observations suggest that the intertrigeminal region receives a convergence of sensory inputs capable of driving apneic responses and that it may represent a common link between input from different portions of the airway and the respiratory neurons that mediate apneic reflexes.

Key words: intertrigeminal region; apnea; respiration; chemical microstimulation; tract tracing; trigeminal
Reflexive apnea is critical for protecting the airways and lungs from potentially damaging events such as aspiration of water or food. The sensory input that causes transient cessation of breathing is conveyed by several cranial nerves, including the ethmoidal branch of the trigeminal nerve, which innervates the nasal mucosa (James and De Burgh Daly, 1972; Yavari et al., 1996), and the branches of the glossopharyngeal and vagal nerves that supply the oropharynx, larynx, and lung (Lucier et al., 1978; Sant'Ambrogio et al., 1995). The first central synapses for these airway sensors lie in the nucleus of the solitary tract and the spinal trigeminal nucleus. However, the central pathways for transmission of sensory input from these nuclei to the respiratory control neurons that ultimately suppress breathing remain undefined.

Earlier studies on the trigeminal apneic reflex emphasized a putative relay in the Kölliker-Fuse nucleus. Early studies on the Kölliker-Fuse nucleus demonstrated respiratory suppressive responses to stimulation with electrical current in cats (Cohen, 1971) or large amounts of glutamate in rats (Lara et al., 1994). Furthermore, injection of large volumes of cobalt solution into the Kölliker-Fuse nucleus was shown to block apneic responses to electrical stimulation of the ethmoidal nerve in rats (Dutschmann and Herbert, 1996).

In contrast, our own earlier microstimulation studies, using

Received April 6, 1998; revised May 18, 1998; accepted May 20, 1998.

This work was supported by National Institutes of Health-U.S. Public Health Service Grant NS22835 and a grant to N.L.C. by the American Lung Association and the Massachusetts Thoracic Society. We thank Dr. L. Hersh for donating the UO95 antisera and Quan Hue Ha and Minh Ha for excellent technical assistance. We thank Dr. Tom Scammell and Amy Malick for helpful comments on this manuscript.

Correspondence should be addressed to Dr. Nancy L. Chamberlin, Department of Neurology, Beth Israel Deaconess Medical Center, Harvard Institutes of Medicine, Room 823, 77 Avenue Louis Pasteur, Boston, MA 02115.

Copyright (C) 1998 Society for Neuroscience $0270-6474 / 98 / 186048-09 \$ 05.00 / 0$ much smaller amounts of glutamate, had found only hyperpneic responses or apneusis in the Kölliker-Fuse nucleus, whereas apneic sites were found in the area just ventral to the Kölliker-Fuse nucleus (Chamberlin and Saper, 1994). These studies suggested that there might be a previously unrecognized cell group in the trigeminal complex that mediated apneic responses. However, no anatomical substrate had previously been identified that would explain how this region would receive airway-related sensory information nor how it would project to the respiratory control system.

To test the possibility of the presence of an apneic nucleus in the trigeminal complex, we combined chemical microstimulation with threshold dosages of glutamate with anterograde and retrograde transport to map the distribution of cells and axonal pathways associated with the apneic sites. In doing so, we have identified a previously unrecognized group of cells in the intertrigeminal region that projects to the ventral respiratory group in the medulla. The intertrigeminal region in turn is innervated by sensory subnuclei that receive vagal and glossopharyngeal as well as trigeminal input from the upper airway. The intertrigeminal region may therefore represent a key relay for a wide range of apneic airway protective reflexes.

\section{MATERIALS AND METHODS}

Chemical microstimulation and injections of biotinylated dextran amines at apneic sites. Thirteen male Sprague Dawley rats (275-325 gm) were obtained from Taconic (Germantown, NY). To prepare animals for surgery, anesthesia was induced with $7 \%$ chloral hydrate $(0.8 \mathrm{ml} / 100 \mathrm{gm}$, i.p.). An endotracheal tube was inserted through the oropharynx and a catheter (PE-50 tubing) was placed into a femoral vein. Anesthesia was subsequently maintained by a continuous intravenous infusion of $\alpha$-chloralose. An acceptable level of anesthesia was gauged by the absence of withdrawal movements in response to a noxious foot pinch.

Rats were placed in a stereotaxic apparatus (David Kopf Instruments) with the incisor bar set at the level of ear bar zero, and a burr hole was 
drilled into the skull to allow access to the left rostral pons. The distal end of the endotracheal tube was attached to a flow of humidified $100 \%$ oxygen via a "Y" connector. Breathing caused the rate of gas flow to fluctuate and these changes were measured by a Fleisch pneumograph with a PT5B pressure transducer. The respiratory flow and stimulus timing signals were amplified by a Grass Polygraph and digitized by a computer equipped with A/D hardware and software (TL-2 and Axotape, Axon Instruments, Foster City, CA). These data were analyzed and displayed with an Apple Macintosh computer with Igor Pro software (Wavemetrics, Lake Oswego, OR). Tidal volume was determined by digital integration of the respiratory flow signal.

Glass micropipettes with an outer tip diameter of 10-20 $\mu \mathrm{m}$ were used to pressure-inject glutamate ( $1 \mathrm{~mm}$ in phosphate buffer), a mixture of glutamate $(500 \mu \mathrm{M})$ and biotinylated dextran amines (BDA) $(5 \%, 3000$ molecular weight; Molecular Probes, Eugene, OR), or a mixture of glutamate, BDA, and cholera toxin B subunit (CTB) (0.07\%; List Biologic, Campbell, CA). All pipettes were held by a stereotaxic manipulator and fitted with a piece of Tygon tubing that was connected to an air pressure injection apparatus. The injected volume was determined $( \pm 1$ $\mathrm{nl}$ ) by measuring the movement of the meniscus within the pipette with an eyepiece reticule in an operating microscope.

The rostral lateral pons was initially explored with 1-3 $\mathrm{nl}$ injections of $1 \mathrm{~mm}$ glutamate. Specifically, glass micropipettes filled with glutamate (1 $\mathrm{mM}$ in phosphate buffer) were positioned by stereotaxic coordinates 4 $\mathrm{mm}$ above ear bar zero. At this site, 1-3 nl of glutamate was pressureinjected and the respiratory responses were observed. The pipette was then advanced ventrally in $100 \mu \mathrm{m}$ intervals as the respiratory responses to glutamate were explored. If no respiratory suppressive response occurred in a given track, the pipette was moved to a different location. When a site was reached where glutamate produced a pause in breathing, the coordinates were noted and the pipette was drawn dorsally out of the brain, but not otherwise moved. Air pressure pulses were applied until the remaining glutamate solution was cleared from the pipette, which was then refilled with a solution of BDA (5-7\%) and glutamate $(0.5-1 \mathrm{mM})$ or, in three cases, a mixture of glutamate, BDA, and CTB. The pipette was then reintroduced into the brain at the predetermined dorsoventral coordinate where $6-9 \mathrm{nl}$ of the mixture was injected. The presence of glutamate in the tracer solution allowed respiratory responses to be recorded during tracer injection, thus physiologically characterizing the injection site. The pipette was removed from the brain, intravascular lines were removed, and the wounds were closed. Rats were extubated and allowed to recover from anesthesia.

After a 3-10 d survival period, rats were deeply anesthetized and perfused through the heart as described previously (Chamberlin and Saper, 1994). The skull was opened and placed in the stereotaxic apparatus to block the brains rostral to the injection site in the same plane as the pipette tracks. The brains and cervical spinal cords were removed and immersed in $20 \%$ sucrose overnight. A sliding microtome was used to cut frozen sections through the brain (four series of $40 \mu \mathrm{m}$ in the coronal plane) and spinal cord (three series of $50 \mu \mathrm{m}$ in the horizontal plane). Sections were stored in PBS containing $0.2 \%$ sodium azide until use.

Biotinylated dextran amine labeling was visualized by incubating the tissue for $1 \mathrm{hr}$ with an avidin-peroxide complex (ABC kit; Vector Laboratories, Burlingame) diluted 1:500 in PBS containing 0.25\% Triton $\mathrm{X}-100$ (PBT). The tissue was rinsed three times for $10 \mathrm{~min}$ in PBS and then stained with $0.05 \%$ diaminobenzidine hydrochloride (DAB; Sigma, St. Louis, MO) and $0.01 \% \mathrm{H}_{2} \mathrm{O}_{2}$ in PBS containing $0.01-0.02 \%$ nickel sulfate and $0.01-0.02 \%$ cobalt chloride.

In some cases sections were subsequently immunostained for choline acetyl transferase (ChAT) as follows. The tissue was incubated overnight at room temperature in rabbit anti-ChAT antiserum (UO95, gift of L. Hersh, University of Kentucky) diluted 1:20,000-100,000 in PBS containing $0.25 \%$ Triton $\mathrm{X}-100$ and $3 \%$ normal goat serum (PGT). Tissue sections were rinsed in PBS three to six times for 10 min each. After successive incubations in biotinylated goat anti-rabbit IgG (Vector; 1:500 in PGT; $2 \mathrm{hr}$ ) and then avidin-biotinylated peroxidase complex (Vector elite ABC kit; 1:500 in PBS; 1-2 hr), sections were reacted in 0.05\% DAB and $0.01 \%$ hydrogen peroxide in PBS.

In cases in which BDA and $\mathrm{CTB}$ were injected together, sections were stained for BDA using nickel/cobalt-enhanced DAB as described above and subsequently immunostained for CTB by incubating overnight in goat anti-CTB (List Biological Laboratories) diluted 1:100,000 in PBT. The tissue was then treated with a mouse monoclonal anti-goat antibody (1:1000; Sigma) and the Vector ABC solution (1:500) for $1 \mathrm{hr}$ each with rinses between incubations. DAB staining was accomplished as described

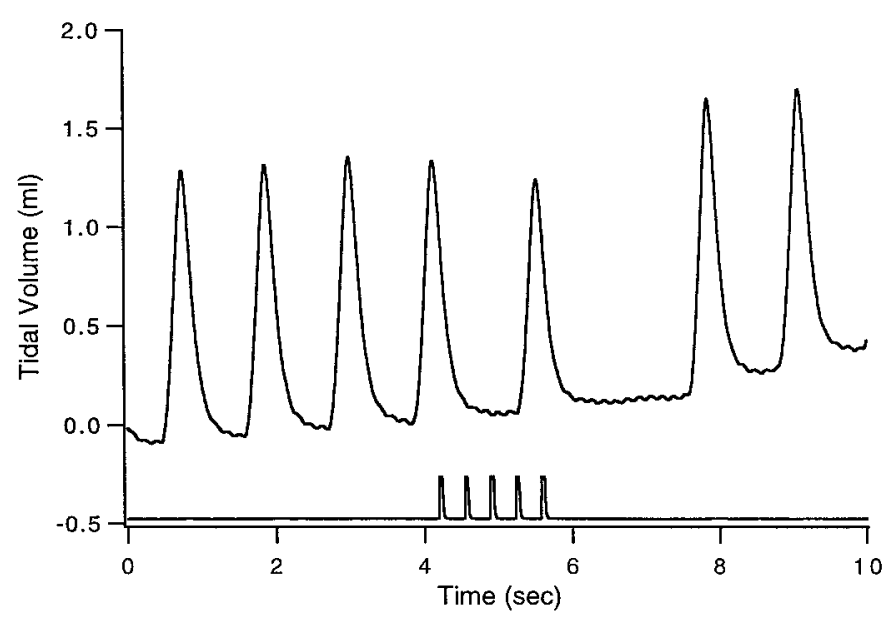

Figure 1. Respiratory suppressive effect of glutamate microinjection. Shown is an example of the effect on breathing of glutamate microinjection in case SPB629. The top trace reflects tidal volume in milliliters. The bottom trace shows the timing of a train of five pressure pulses that ejected $\sim 1.5 \mathrm{nl}$ each of $0.5 \mathrm{~mm}$ glutamate. Note that the first breath after glutamate injection is reduced in amplitude and followed by a pause. The baseline drift is artifactual. In this case the injection site was located just medial to the ventral border of the principal sensory trigeminal nucleus (Fig. 2D, vertical arrow), and the distribution of fibers is shown in Figure 3.

above in the absence of $\mathrm{Ni}$ or $\mathrm{Co}$ ions such that a brown reaction product was formed in CTB-containing neurons. Tissue was mounted on gelatincoated glass microscope slides, dehydrated in graded alcohols, cleared in xylene, and coverslipped with Permaslip mounting medium. After drawings were made and photographs taken (see below), the slides were replaced in xylene to remove the coverslips, and the tissue was counterstained for Nissl substance with thionin $(0.125 \%)$.

Retrograde tracing with horseradish peroxidase conjugated to wheat germ agglutinin. Injections of 3-6 $\mathrm{nl}$ of a 1\% solution of horseradish peroxidase conjugated to wheat germ agglutinin (WGA-HRP) (Sigma) were pressure-injected into the ventrolateral medulla in eight additional cases. After $30-60 \mathrm{hr}$ survival, the animals were reanesthetized and perfused as described above except that the fixative contained 0.5 or $1 \%$ paraformaldehyde and $1.25 \%$ glutaraldehyde. The brains were removed and immersed overnight in $20 \%$ sucrose, and frozen sections were cut into three series of $50 \mu \mathrm{m}$. One series was processed according to the tetramethylbenzidine (TMB) method of de Olmos and coworkers (1978). Sections were then mounted on gelatin-coated slides, air-dried, rapidly counterstained with thionin, dehydrated, cleared in xylene, and coverslipped. The staining and dehydration were performed with all solutions kept at $\sim 8^{\circ} \mathrm{C}$ to prevent loss of the HRP/TMB reaction product.

Data analysis. The distribution of labeled cells and axon terminals was mapped with a microscope equipped with a camera lucida drawing tube. Line drawings were digitized with a flatbed scanner, and "Canvas" (Deneba) software was used to create the final maps of injection sites, anterogradely labeled axon terminals, and retrogradely labeled cells. Digital photomicrographs were taken with a Kodak 460 DCS camera mounted on a Zeiss microscope and were prepared using Adobe Photoshop on an Apple Macintosh computer. Images were sharpened, brightness and contrast were adjusted, and then images were printed with a Kodak 8600 dye sublimation printer.

\section{RESULTS}

\section{Anatomical location of respiratory suppressive effects of pontine stimulation}

Microinjection of doses of glutamate as low as 1 pmol caused abrupt, transient inhibition of inspiration, which we term hypopnea or apnea (Fig. 1). In previous studies (Chamberlin and Saper, 1994) we reserved the term apnea for pauses in breathing that exceeded $5 \mathrm{sec}$. In this study we reduced the dose of glutamate to enhance the anatomical resolution of our mapping studies. Thus the apnea durations were shorter. Nonetheless, because the re- 

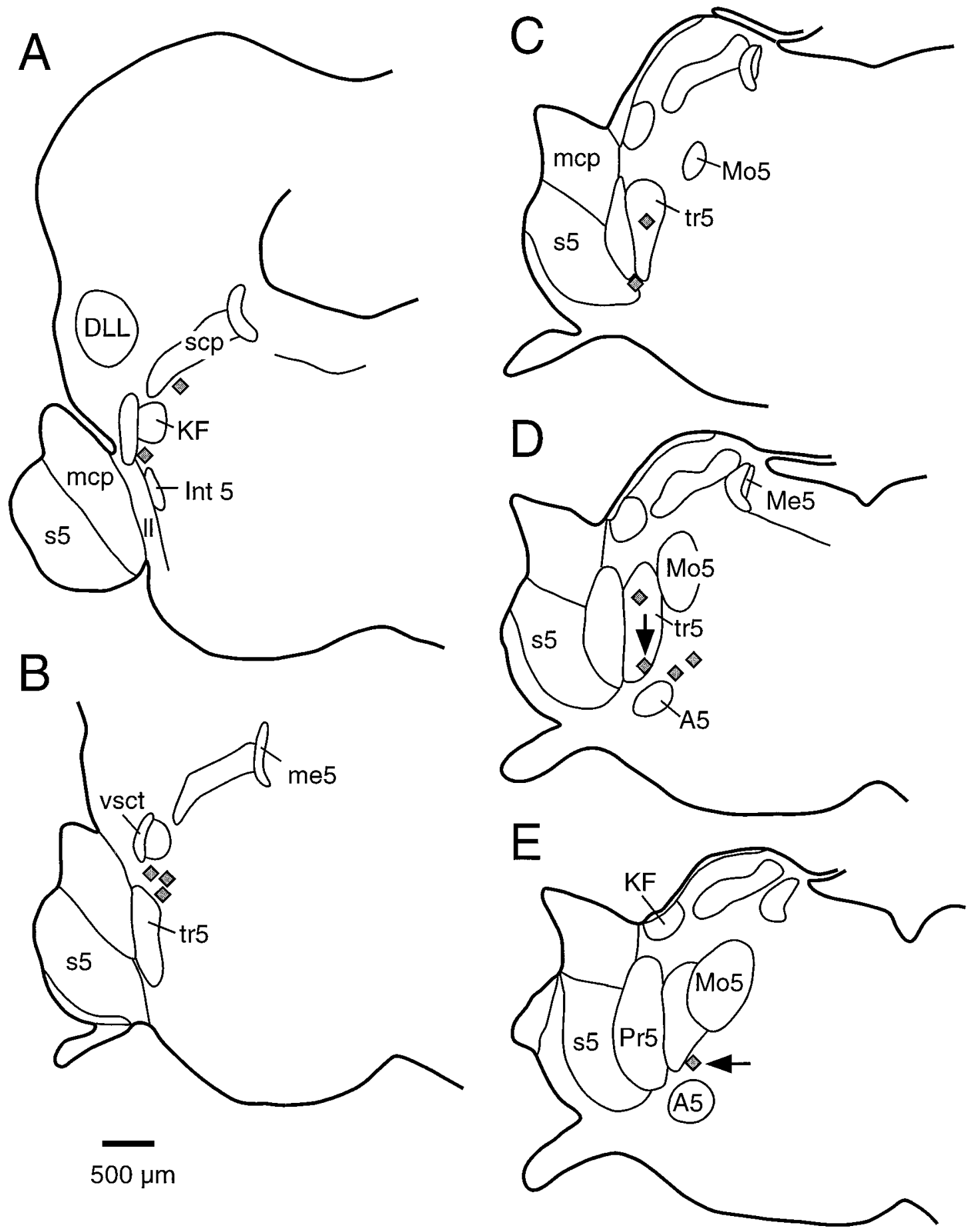

Figure 2. Anatomical distribution of hypopneic sites. Each gray diamond represents the location where microinjection of glutamate and BDA produced a decrease in respiratory rate in one rat. $A-E$, Representative coronal sections through the rat brain at $160 \mu \mathrm{m}$ intervals. The vertical arrow in $D$ shows the case in which the response is shown in Figure 1. The horizontal arrow in $E$ shows the case that is also presented in Figure 5. DLL, Dorsal nucleus of the lateral lemniscus; Int 5, intertrigeminal nucleus; $K F$, Kölliker-Fuse nucleus; $l l$, lateral lemniscus; $m c p$, middle cerebellar peduncle; $M e 5$, mesencephalic trigeminal nucleus, $m e 5$, mesencephalic trigeminal tract; Mo5, motor trigeminal nucleus; Pr5, principal sensory trigeminal nucleus; $s 5$, sensory root of the trigeminal nerve; scp, superior cerebellar peduncle; tr5, motor roots; $v s c t$, ventral spinocerebellar tract.

sponse that we observed in this study was a consistent increase in expiratory time, we use the terms apnea and hypopnea interchangeably to refer to effects on breathing manifested as a transient decrease in respiratory rate with or without a concomitant decrease in tidal volume.

The anatomical distribution of injection sites where glutamate caused hypopnea is summarized in Figure 2. In general, nearly all apneic sites were found among the fiber bundles between the motor and principal sensory trigeminal nuclei. These fibers, which primarily represent the motor rootlets of the trigeminal nerve, contain scattered medium- to large-sized multipolar neurons, and the entire area has been termed the intertrigeminal region. Ros- 


\begin{tabular}{|c|c|c|c|c|c|c|c|c|c|c|c|c|}
\hline & SPB576 & SPB579 & SPB592 & SPB598 & SPB601 & SPB609 & SPB611 & SPB614 & SPB629 & SPB635 & SPB636 & SPB639 \\
\hline Transport & + & +++ & ++ & +++ & + & + & +++ & +++ & +++ & +++ & +++ & ++ \\
\hline KF & no & no & no & ++ & + & no & + & + & no & ++ & + & no \\
\hline HMN (12) & no & no & no & + & no & no & ++ & no & no & +++ & no & no \\
\hline NTS & no & no & no & ++ & no & no & no & ++ & no & ++ & no & no \\
\hline FMN (7) & no & +++ & no & +++ & no & + & ++ & +++ & ++ & +++ & ++ & ++ \\
\hline Bötz. c. & + & +++ & ++ & +++ & + & + & +++ & +++ & +++ & +++ & +++ & ++ \\
\hline rVRG & + & +++ & ++ & +++ & + & + & +++ & +++ & ++ & +++ & +++ & ++ \\
\hline $\mathrm{cVRG}$ & + & +++ & + & +++ & no & + & +++ & +++ & ++ & +++ & +++ & ++ \\
\hline Oculomotor & + & + & no & + & no & no & no & no & ++ & ++ & + & + \\
\hline Superior colliculus & no & no & + & + & no & no & no & + & ++ & +++ & ++ & no \\
\hline
\end{tabular}

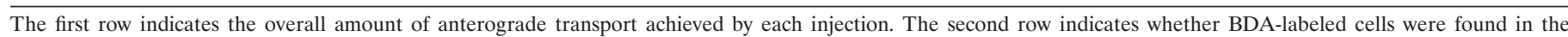

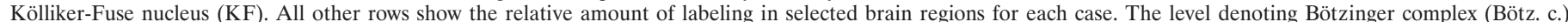

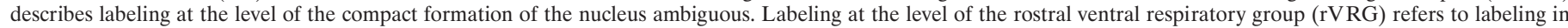

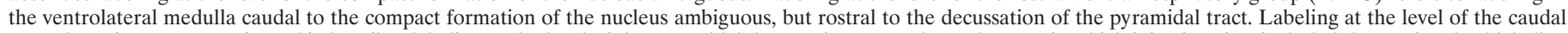

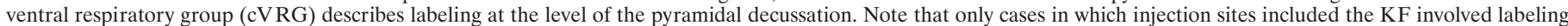
of the nucleus of the solitary tract or the hypoglossal motor nucleus [HMN (12)].

trally, apneic sites stretched up to the ventral border of the Kölliker-Fuse nucleus (Fig. 2A,B) but were never found within this nucleus (Chamberlin and Saper, 1994). Caudally, apneic sites stretched ventrally along the motor trigeminal rootlets into the A5 region (Fig. 2D,E).

\section{Projections from apneic sites: anterograde tracing with BDA}

In 12 cases in which injection of a mixture of glutamate and BDA produced a hypopneic response, anterogradely labeled fibers were traced from the injection site to their terminal fields. Although these injection sites were widely varied in their location (Fig. 2), all demonstrated a consistent pattern of efferent projections. Labeled fibers descended in the ventrolateral region of the brainstem just medial to the exit of the seventh nerve at pontine levels and medial to the ventromedial edge of the spinal trigeminal nucleus in the medulla. Table 1 summarizes the brain regions in which labeled axon terminals were found in each case. In all cases terminals were seen bilaterally, with a strong ipsilateral preference, in the ventrolateral quadrant of the medulla. In most cases terminals were found in the lateral portions of the facial motor nucleus. The heaviest labeling was always seen just caudal to this area at the level of the compact formation of the nucleus ambiguus in the periambigual region (Figs. 3, 4A). Examination of tissue stained for ChAT as well as anterogradely labeled terminals showed that although axon terminals were located in the vicinity of the nucleus ambiguus, they rarely formed close appositions with cholinergic neurons (Fig. 3). The distribution of medullary terminals in a typical case, SPB629, is shown in Figure 4 , where it can be seen that terminals were found in the entire length of the ventrolateral medulla from the periambigual region to the spinomedullary junction. In several cases large BDA injections also produced sparse retrograde labeling. These cells were found in the ventrolateral medulla in the same area as the labeled terminals and in the spinal trigeminal nucleus. In the cases in which BDA injection sites included a few labeled cells in the Kölliker-Fuse nucleus, labeled axon terminals were also found in the nucleus of the solitary tract and in the hypoglossal motor nucleus. Efferent fibers were not found in the dorsal vagal complex or the hypoglossal nucleus in the cases in which injections did not include the Kölliker-Fuse nucleus. In several cases labeled axons were seen in the white matter of the ipsilateral cervical

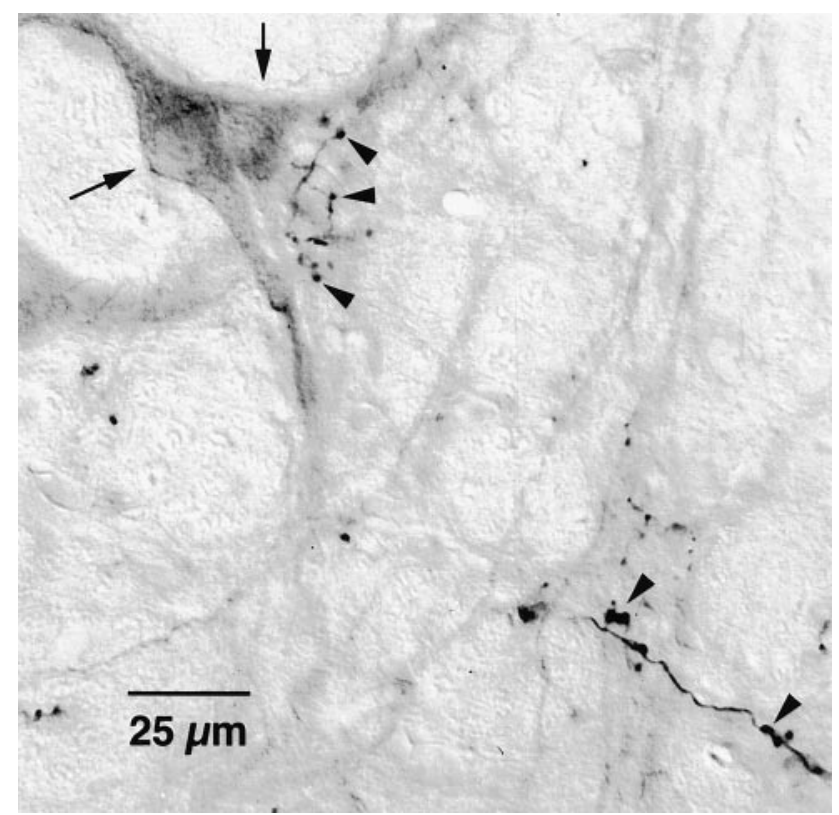

Figure 3. Projections of apneic sites to the ventrolateral medulla. Photomicrographs showing BDA-labeled terminals (arrowheads) and ChATimmunoreactive neurons (arrows). Note that terminals are nearby but not closely apposed to ChAT-immunoreactive neurons.

spinal cord; however, no labeled terminals were seen in the phrenic motor nucleus.

Forebrain projections from apneic sites were less consistent and less dense than medullary terminal fields. Fibers were seen to cross the pontine tegmentum between the decussation of the superior cerebellar peduncle and the base of the pons. Many axons turned dorsally near the red nucleus, and in most cases labeled axon terminals were seen in the contralateral oculomotor nucleus. Other axons continued rostrally in the central tegmental tract and in some cases formed terminals in the deep layers of the superior colliculus. Fibers continued rostrally in the central tegmental field and in the medial lemniscus. A few terminals were found in the prerubral field, the zona inserta, and the ventromedial nucleus of the thalamus. 

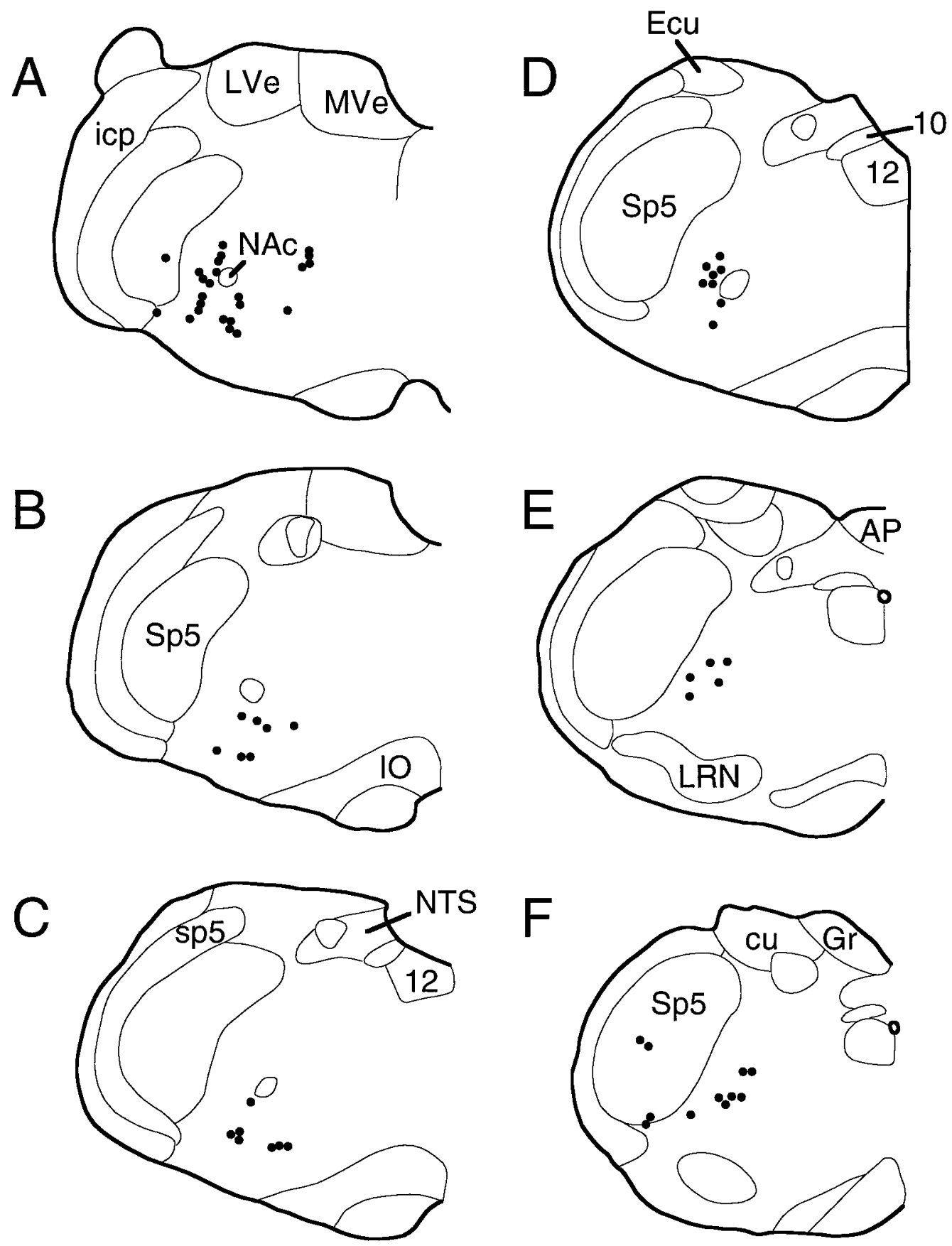

Figure 4. Distribution of terminal fields from a hypopneic site. Each dot represents three to eight BDA-labeled axon terminals. Labeled fibers of passage are not shown. Each drawing $(A-F$, rostral to caudal) is a representative coronal section through the medulla separated by $\sim 160 \mu \mathrm{m}$ intervals. Note that labeling is heaviest at rostral levels and extends the entire length of the medulla (case SPB629). 10, Dorsal motor vagal nucleus; 12, hypoglossal motor nucleus; $A P$, area postrema; $c u$, cuneate fasciculus; $E c u$, external cuneate nucleus; $G r$, gracile nucleus; $i c p$, inferior cerebellar peduncle; $I O$, inferior olive; $L V e$, lateral vestibular nucleus; $L R N$, lateral reticular nucleus; $M V e$, medial vestibular nucleus; NAc, compact formation of the nucleus ambiguus; $N T S$, nucleus of the solitary tract; $s p 5$, spinal trigeminal tract; $S p 5$, spinal trigeminal nucleus.

\section{The location of apneic neurons: retrograde tracing with WGA-HRP}

To identify the neurons of origin of the observed medullary projections from apneic sites, injections of WGA-HRP were placed into the ventrolateral medulla at different rostrocaudal levels in eight rats. In each of these cases neurons were identified at nonapneic sites in the Kölliker-Fuse nucleus and the lateral crescent parabrachial subnucleus, as described previously (Fig. 5)
(Herbert et al., 1990; Chamberlin and Saper, 1992). In addition, a population of medium-sized triangular, spindle-shaped, or multipolar neurons was found in the intertrigeminal region whose distribution matched that of the apneic sites mapped in the microstimulation experiments (Fig. 5). Specifically, a cluster of retrogradely labeled neurons was seen stretching from just ventral to the Kölliker-Fuse nucleus at rostral levels of the motor trigeminal nucleus through the region between the principal sensory and 


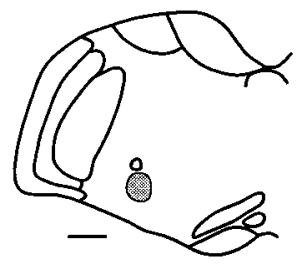

A

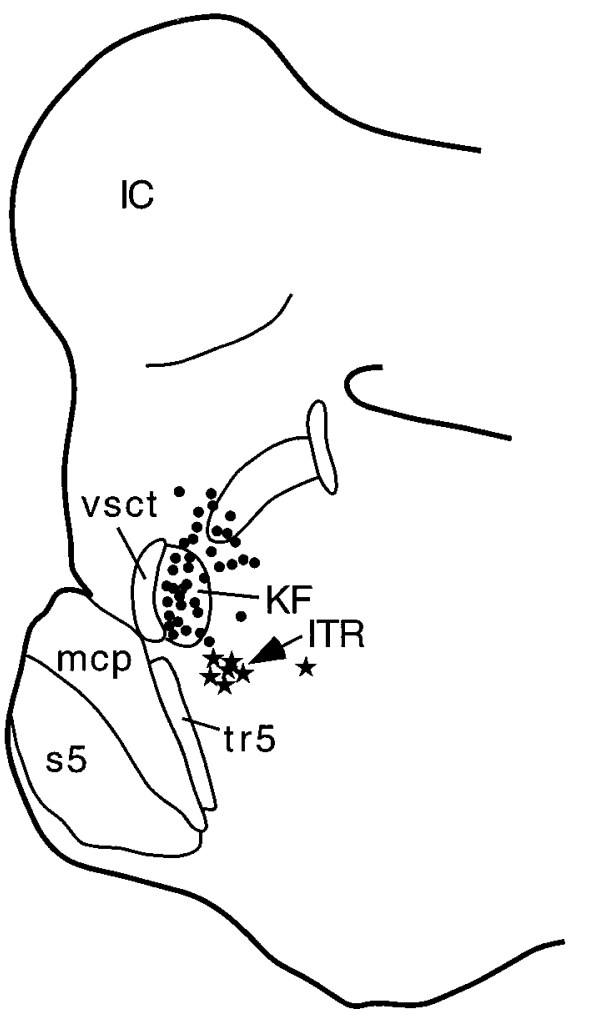

B

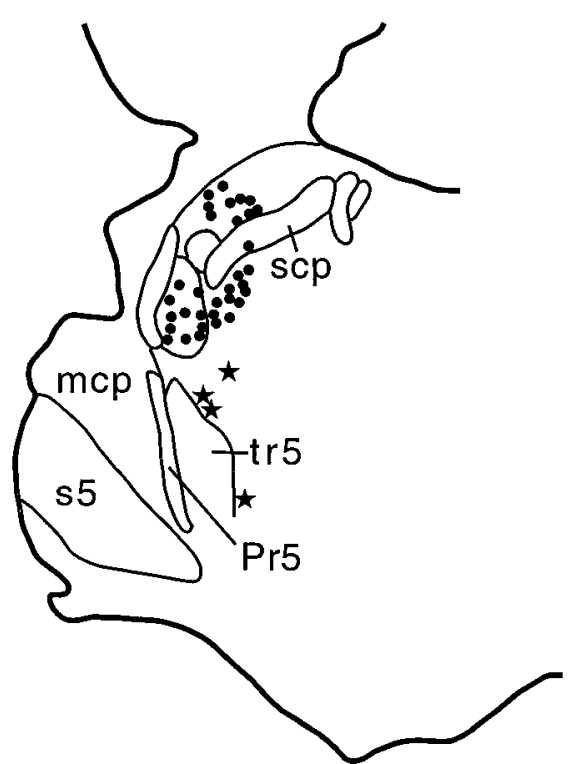

C

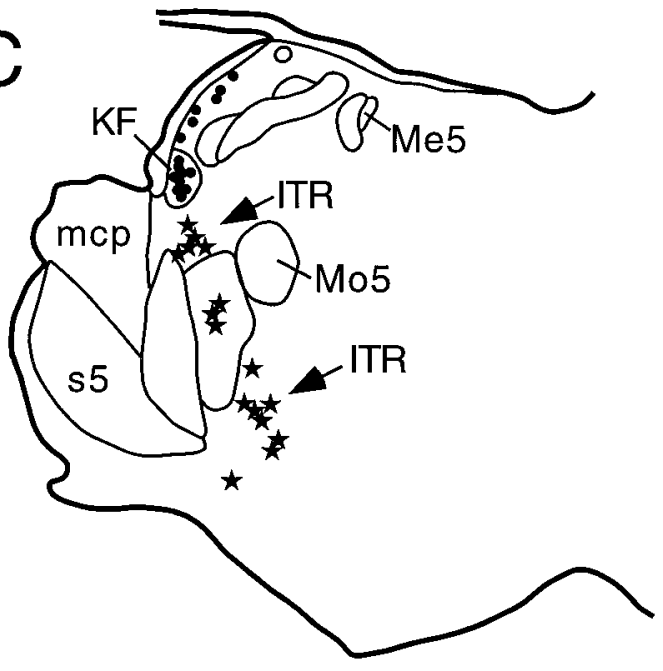

D

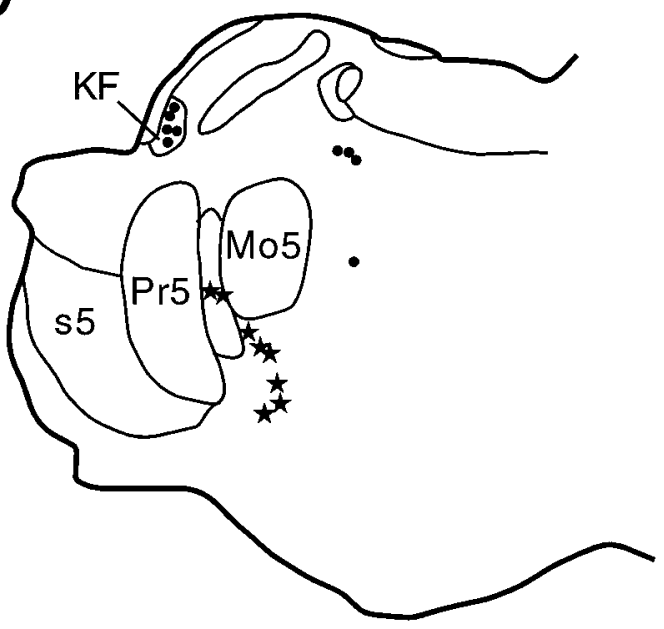

E

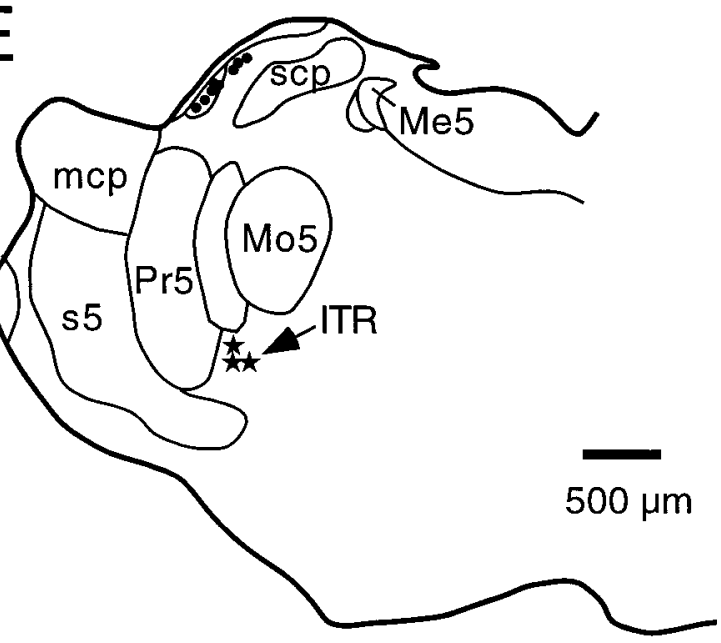

Figure 5. Distribution of pontine neurons projecting to the rostral ventrolateral medulla. $A-E$, Coronal sections (rostral to caudal) through the pons depicting the locations of neurons retrogradely labeled after an injection of WGA-HRP into the rostral ventrolateral medulla just ventral to the compact formation of the nucleus ambiguus (inset: scale bar, $500 \mu \mathrm{m})$. The retrogradely labeled neurons in the intertrigeminal region (ITR) are shown as stars. Note that these cells extend from the area just ventral to the Kölliker-Fuse nucleus, stretching ventrally and caudally underneath the motor trigeminal nucleus. Abbreviations are as in Figure 2. 
Figure 6. Distribution of retrogradely labeled neurons from apneic sites. In this case (SPB639), cholera toxin subunit $\mathrm{B}(0.07 \%)$ was included in the injection mixture. In $A-D$, (rostral to caudal) coronal sections through the medulla, each dot represents a single retrogradely labeled neuron.
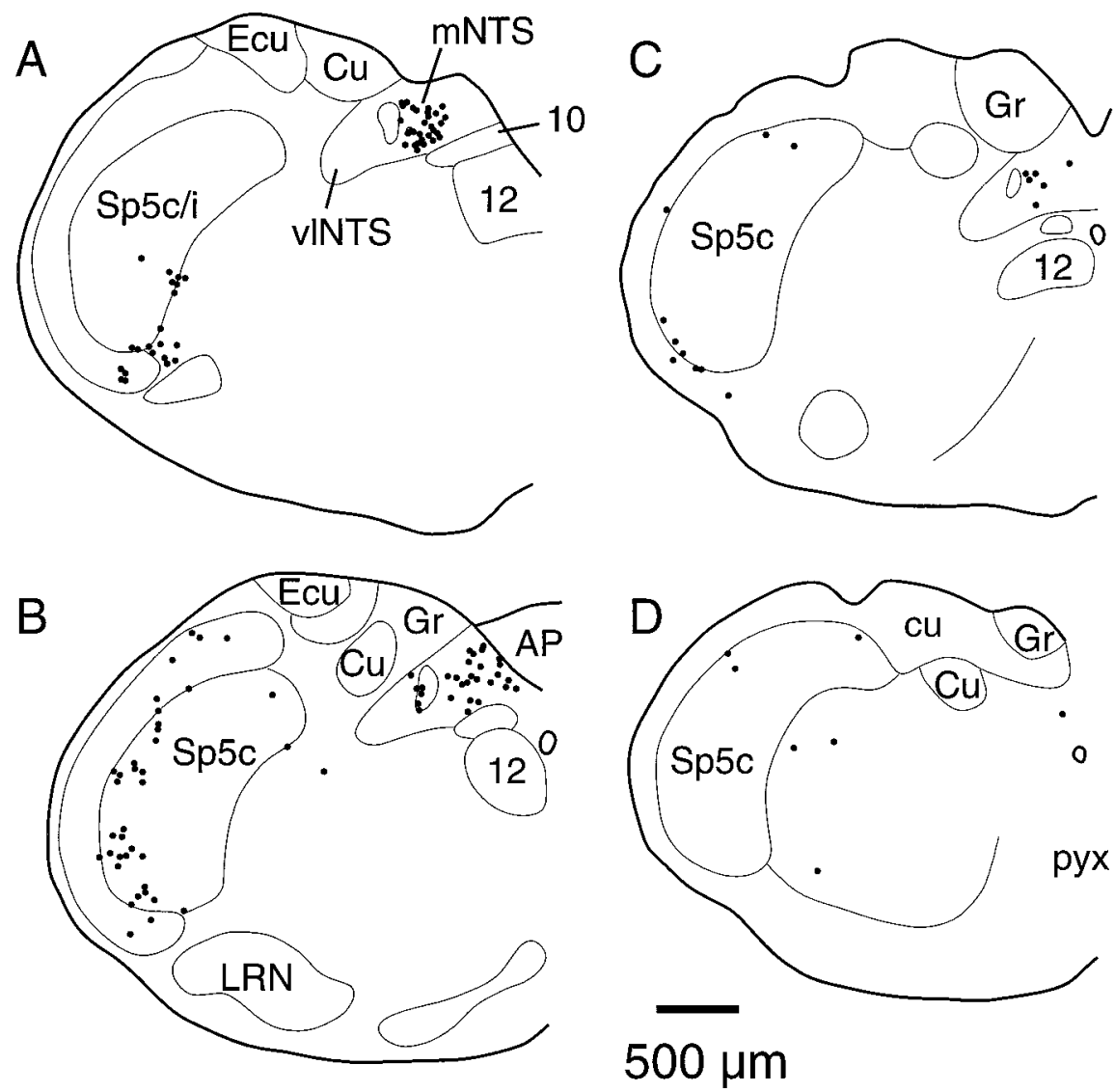

motor trigeminal nuclei, into the area ventral to the motor trigeminal nucleus.

\section{Inputs to apneic sites: retrograde labeling with CTB}

In three cases (SPB635, SPB636, and SPB639) CTB was included in the mixture of glutamate and BDA that was injected at an apneic site. In each case retrogradely labeled neurons were found in the spinal trigeminal nucleus and in the nucleus of the solitary tract (Fig. 6). In the spinal trigeminal nucleus, the greatest number of labeled neurons was found in the transition region between its caudalis and interpolaris divisions. In each of these cases, retrogradely labeled neurons were also located in the interstitial (Fig. 6B) and medial (Fig. 6A-C) subnuclei of the nucleus of the solitary tract. Very few retrogradely labeled neurons were seen in the Kölliker-Fuse nucleus, parabrachial complex, or pontomedullary reticular formation.

\section{DISCUSSION}

We identified a population of neurons in the intertrigeminal region of the pons from which apneic responses can be elicited and which project to the ventral respiratory group. Intertrigeminal apneic sites receive inputs from the nucleus of the solitary tract as well as the spinal trigeminal nucleus. We suggest that the intertrigeminal region may participate in trigeminal, vagal, or glossopharyngeal apneic reflexes. Figure 7 illustrates proposed pathways for these reflexes.

\section{Technical considerations}

Combining glutamate with tracers enabled measurement of respiratory responses at the very sites at which tracing originated, a powerful technique for correlating function with neuronal con- nectivity. Our use of threshold doses of glutamate provided the finest anatomical resolution possible with microstimulation mapping. Although there is no certainty that the neurons producing the response and those that transport the tracer are identical, the validity of the results requires only an overlap between these two populations. Cells that do not mediate apneic responses may be labeled in some cases, but the labeling of irrelevant terminal fields may vary from case to case, whereas those pathways crucial for the response should be labeled in all cases. Therefore, examination of a number of cases should reveal the relevant projections. Without exception, every injection at an apneic site labeled terminals in the ventral respiratory group in the medulla (Ellenberger and Feldman, 1990). In contrast, projections to other targets, which are thought to be irrelevant to the respiratory responses (e.g., the oculomotor nucleus), were labeled in only a fraction of cases.

The tracers that we used (BDA, CTB, WGA-HRP) are capable of bidirectional transport, which can complicate the interpretation of results. Our BDA injections were quite small and produced very few retrogradely labeled cells. Hence it is unlikely that axonal transport represented collaterals of retrogradely labeled neurons. WGA-HRP cases were used only to identify retrograde transport. The intertrigeminal region, into which we injected $\mathrm{CTB}$, is crossed by nearly all of the connections of the parabrachial complex with the spinal cord and the medulla. However, CTB is not taken up by uninjured fibers of passage, and our injection method, using fine micropipettes, did not damage this region, as witnessed by the lack of retrograde labeling in the parabrachial complex in these cases. Hence the retrograde label seen represents input to the intertrigeminal region neurons. This conclusion was confirmed by examining material from earlier 


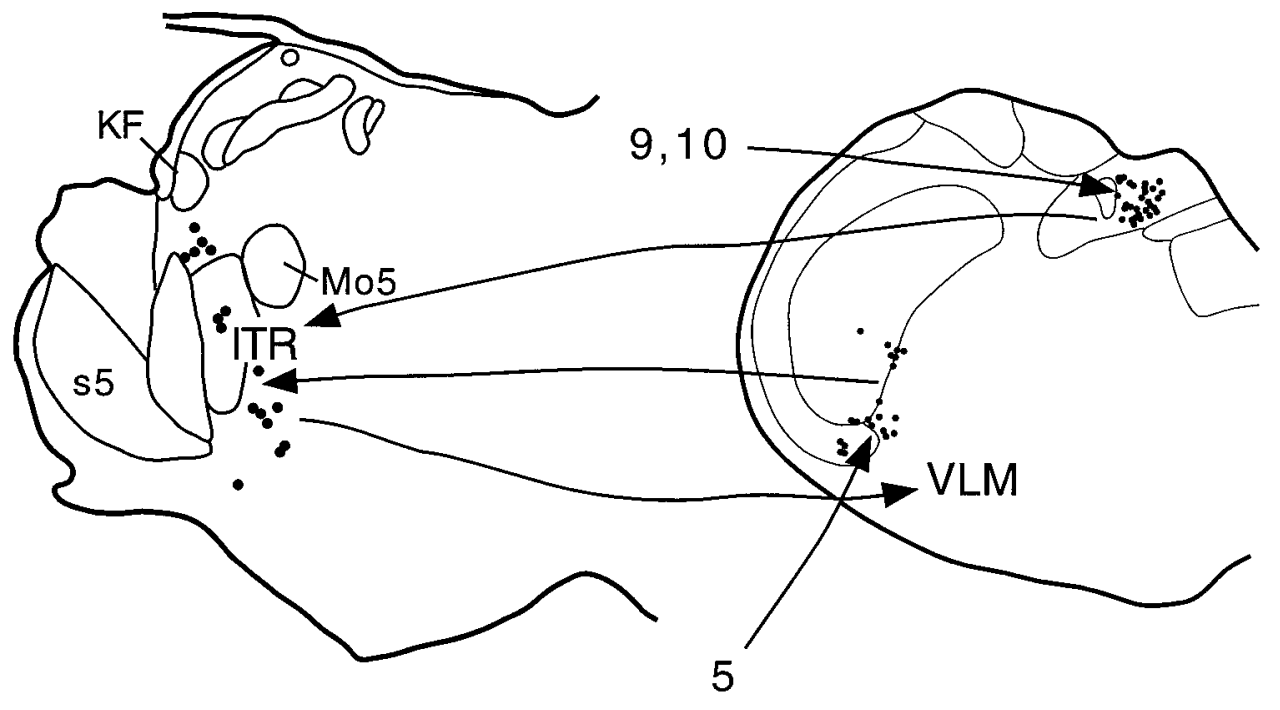

Figure 7. Summary diagram illustrating the proposed apneic pathways. Sensory information from the upper airway carried by the trigeminal nerve (5) terminates in the spinal trigeminal nucleus, which in turn projects to the intertrigeminal region (ITR). Vagal (10) and glossopharyngeal (9) afferents terminate in the nucleus of the solitary tract, which also projects to the ITR. The ITR causes apnea via a projection to the ventral respiratory group in the ventrolateral medulla $(V L M) . K F$, Kölliker-Fuse nucleus; Mo5, motor trigeminal nucleus; $s 5$, sensory root of the trigeminal nerve. studies in which we injected PHA-L into these ascending pathways (Herbert et al., 1990).

Previous investigations using microinjections of excitatory amino acids or blockers of synaptic transmission implicated the Kölliker-Fuse nucleus as an apneic region (Lara et al., 1994; Dutschmann and Herbert, 1996). In contrast, our microstimulation studies (Chamberlin and Saper, 1994) have shown that activation of neurons in the Kölliker-Fuse nucleus produces inspiratory facilitation manifested as hyperpnea or apneusis. Discrepancies between these results may be accounted for by the volumes of chemicals used in different studies. We found that doses of glutamate as low as $1 \mathrm{nl}$ of a $1 \mathrm{~mm}$ solution caused hypopnea when injected into the intertrigeminal region, indicating that activation of only a few neurons may be sufficient to affect the respiratory rhythm. By contrast, reported apneic sites in the Kölliker-Fuse nucleus were obtained from injections ranging from 20 to $30 \mathrm{nl}$ that contained $10-100 \mathrm{~mm}$ concentrations of glutamate (Lara et al., 1994). These comparatively large doses, although centered in the Kölliker-Fuse nucleus, may have spread into the adjacent intertrigeminal region. Likewise, Dutschmann and Herbert (1996) demonstrated interruption of apneic effects of ethmoidal nerve stimulation by injecting large volumes (50-150 nl) of cobalt chloride, which may have spread and also blocked synaptic transmission in neighboring brain areas. Because they did not provide control injections into the area just ventral to the Kölliker-Fuse nucleus, where we found apneic responses, it is possible that their cobalt injections attenuated ethmoidal nerve apneic responses by blocking neurotransmission in the intertrigeminal region.

\section{Anatomical considerations}

The area between the principal sensory and motor trigeminal nuclei has been referred to as the intertrigeminal region (Brodal, 1981). Cells of this region are intermingled among axons that consist largely, although not exclusively, of motor trigeminal fibers. This region is different from a cell group previously termed "the intertrigeminal nucleus," which comprises accessory trigeminal motor neurons that innervate the tensor tympani muscle (Spangler et al., 1982; Jacquin et al., 1983). By contrast, cells in the intertrigeminal region are not cholinergic, nor are they labeled by tracer injections into the motor trigeminal nerve (Jacquin et al., 1983).
Neurons in the intertrigeminal region have been retrogradely labeled by tracer injections into the superior colliculus and the oculomotor nucleus (Guerra-Seijas et al., 1993; Yasui et al., 1993). Interestingly, sites in the ventral part of the intertrigeminal region have been associated with the blink reflex (Holstege et al., 1986), a response that we occasionally encountered in our animals. These observations raise the possibility that the intertrigeminal region may be more broadly involved in facial protective reflexes.

All of our cases demonstrated a projection from intertrigeminal apneic sites to the ventrolateral medulla (Fig. 4, Table 1). Because the ventrolateral medulla contains the ventral respiratory group, a complex of neurons that generate respiratory rhythm and drive the respiratory motor neurons, we presume that the intertrigeminal apneic response is produced by alterations in the activity of some of these neurons. Efferent projections from the intertrigeminal region were found along the entire rostrocaudal extent of the ventrolateral medulla in nearly all cases, so it is not clear which subsets of respiratory neurons mediate the pontine apneic response. However, in most cases the densest terminal labeling was seen at rostral levels of the ventrolateral medulla, coextensive with the Bötzinger and pre-Bötzinger complexes (Ellenberger and Feldman, 1990; Smith et al., 1991). The latter region, which has been hypothesized to contain the respiratory rhythmgenerating neurons (Smith et al., 1991), seems to be the most likely candidate for orchestrating pauses in breathing that reset the rhythm pattern.

\section{Physiological role of the intertrigeminal apneic response}

Studies in muskrat, rat, and dog have shown that apnea can be evoked by noxious stimulation of the nasal mucosa (James and De Burgh Daly, 1972; Dutschmann and Herbert, 1996; Yavari et al., 1996). This apneic reflex is mediated by the ethmoidal branch of the trigeminal nerve and can be blocked by cobalt injections into the rostral pons (Dutschmann and Herbert, 1996). Neurons in the intertrigeminal region may mediate sensory apnea by a direct input from trigeminal primary afferents (Panneton, 1991). However, ethmoidal nerve-evoked apnea can also be blocked by lidocaine or kynurenate injections into the spinal trigeminal nucleus, suggesting that the primary afferents triggering this response terminate in the medulla (Panneton and Yavari, 1995). Intrigu- 
ingly, we found that the intertrigeminal region receives input from the same part of the spinal trigeminal nucleus (the ventral region at the caudalis/interpolaris transition zone) at which nasal apneic responses can be blocked (compare Fig. 6 with Panneton and Yavari, 1995, their Figs. 2, 4, and 7).

We also found that apneic sites in the intertrigeminal region received inputs from the nucleus of the solitary tract, mainly from its medial and interstitial subnuclei (Fig. 6), which receive vagal and glossopharyngeal primary afferents innervating the lungs and upper airway (Kalia and Richter, 1988; Altschuler et al., 1989; Patrickson et al., 1991; Furusawa et al., 1996). Therefore, information from the soft palate, pharynx, larynx, and lungs may reach the intertrigeminal region via a relay in the nucleus of the solitary tract.

The connections between the nucleus of the solitary tract and the intertrigeminal region may mediate the apnea that occurs during swallowing and pulmonary stretch or after mechanical or chemical upper airway stimulation. A projection from neurons in the interstitial subnucleus of the nucleus of the solitary tract that are active during swallowing (Ootani et al., 1995) to the intertrigeminal region may be responsible for the transient apnea that is essential to avoid aspiration of food or liquids before they can be swallowed. Apnea mediated by the superior laryngeal branch of the vagus nerve, which is induced by activation of laryngeal receptors (Sant'Ambrogio et al., 1991), may also rely on the intertrigeminal region. Finally, lung inflation resulting in pulmonary stretch causes the well known Hering-Breuer apneic reflex. Neurons mediating this vagal reflex have been localized to a region of the nucleus of the solitary tract just caudal to the obex and medial to the solitary tract (Bonham and McCrimmon, 1990). The finding of retrogradely labeled neurons in this region after injections into intertrigeminal apneic sites supports the hypothesis that pulmonary stretch information may reach respiratory control neurons via a relay in the intertrigeminal region. Alternatively, the intertrigeminal region may play a modulatory role in the Hering-Breuer reflex. The increased expiratory time after intertrigeminal microstimulation may reflect potentiation of the Hering-Breuer response. Thus, the intertrigeminal region may be the penultimate nucleus in several different pathways that lead to the ventrolateral medulla for eliciting apnea during swallowing or after stimulation of the upper airway or the lung (Fig. 7).

\section{REFERENCES}

Altschuler SM, Bao X, Bieger D, Hopkins DA, Miselis RR (1989) Viscerotopic representation of the upper alimentary tract in the rat: sensory ganglia and nucleus of the solitary and spinal trigeminal tracts. J Comp Neurol 283:248-268.

Bonham AC, McCrimmon DR (1990) Neurones in a discrete region of the nucleus tractus solitarius are required for the Breuer-Hering reflex in rat. J Physiol (Lond) 427:261-280.

Brodal A (1981) Neurological anatomy. In relation to clinical medicine. Oxford: Oxford UP.

Chamberlin NL, Saper CB (1992) Topographic organization of cardiovascular responses to electrical and glutamate microstimulation of the parabrachial nucleus in the rat. J Comp Neurol 326:245-262.

Chamberlin NL, Saper CB (1994) Topographic organization of respiratory responses to glutamate microstimulation of the parabrachial nucleus in the rat. J Neurosci 14:6500-6510.

Cohen MI (1971) Switching of the respiratory phases and evoked phrenic responses produced by rostral pontine electrical stimulation. J Physiol (Lond) 217:133-158. de Olmos J, Hardy H, Heimer L (1978) The afferent connections of the main and the accessory olfactory bulb formations in the rat: an experimental HRP-study. J Comp Neurol 181:213-244.

Dutschmann M, Herbert H (1996) The Kölliker-Fuse nucleus mediates the trigeminally induced apnoea in the rat. NeuroReport 7:1432-1436.

Ellenberger HH, Feldman JL (1990) Subnuclear organization of the lateral tegmental field of the rat. I: Nucleus ambiguus and ventral respiratory group. J Comp Neurol 294:202-211.

Furusawa K, Yasuda K, Okuda D, Tanaka M, Yamaoka M (1996) Central distribution and peripheral functional properties of afferent and efferent components of the superior laryngeal nerve: morphological and electrophysiological studies in the rat. J Comp Neurol 375:147-156.

Guerra-Seijas MJ, Labandeira Garcia J, Tobio J, Gonzalez F (1993) Neurons located in the trigeminal sensory complex and the lateral pontine tegmentum project to the oculomotor nucleus in the rabbit. Brain Res 601:1-13.

Herbert H, Moga MM, Saper CB (1990) Connections of the parabrachial nucleus with the nucleus of the solitary tract and the medullary reticular formation in the rat. J Comp Neurol 293:540-580.

Holstege G, Tan J, van Ham JJ, Graveland GA (1986) Anatomical observations on the afferent projections to the retractor bulbi motoneuronal cell group and other pathways possibly related to the blink reflex in the cat. Brain Res 374:321-334.

Jacquin MF, Rhoades RW, Enfiejian HL, Egger MD (1983) Organization and morphology of masticatory neurons in the rat: a retrograde HRP study. J Comp Neurol 218:239-256.

James JE, De Burgh Daly M (1972) Reflex respiratory and cardiovascular effects of stimulation of receptors in the nose of the dog. J Physiol (Lond) 220:673-696.

Kalia M, Richter D (1988) Rapidly adapting pulmonary receptor afferents: I. Arborization in the nucleus of the tractus solitarius. J Comp Neurol 274:560-573.

Lara JP, Parkes MJ, Silva-Carvhalo L, Izzo P, Dawid-Milner MS, Spyer KM (1994) Cardiovascular and respiratory effects of stimulation of cell bodies of the parabrachial nuclei in the anaesthetized rat. J Physiol (Lond) 477:321-329.

Lucier GE, Daynes J, Sessle BJ (1978) Laryngeal reflex regulation: peripheral and central neural analyses. Exp Neurol 62:200-213.

Ootani S, Umezaki T, Shin T, Murata Y (1995) Convergence of afferents from the SLN and GPN in cat medullary swallowing neurons. Brain Res Bull 37:397-404.

Panneton WM (1991) Primary afferent projections from the upper respiratory tract in the muskrat. J Comp Neurol 308:51-65.

Panneton WM, Yavari P (1995) A medullary dorsal horn relay for the cardiorespiratory responses evoked by stimulation of the nasal mucosa in the muskrat Ondatra zibethicus: evidence for excitatory amino acid transmission. Brain Res 691:37-45.

Patrickson JW, Smith TE, Zhou S-S (1991) Afferent projections of the superior and recurrent laryngeal nerves. Brain Res 539:169-174.

Sant'Ambrogio G, Anderson JW, Sant'Ambrogio FB, Mathew OP (1991) Response of laryngeal receptors to water solutions of different osmolality and ionic composition. Respir Med 85:57-60.

Sant'Ambrogio G, Tsubone H, Sant'Ambrogio FB (1995) Sensory information from the upper airway: role in the control of breathing. Respir Physiol 102:1-16.

Smith JC, Ellenberger HH, Ballanyi K, Richter DW, Feldman JL (1991) Pre-Botzinger complex: a brainstem region that may generate respiratory rhythm in mammals. Science 254:726-729.

Spangler KM, Henkel CK, Miller Jr IJ (1982) Localization of the motor neurons to the tensor tympani muscle. Neurosci Lett 32:23-27.

Yasui Y, Kayahara T, Shiroyama T, Nakano K (1993) Neurons in the intertrigeminal region of the rat send projection fibers to the superior colliculus. Neurosci Lett 159:39-42.

Yavari P, McCulloch PF, Panneton WM (1996) Trigeminally-mediated alteration of cardiorespiratory rhythms during nasal application of carbon dioxide in the rat. J Auton Nerv Syst 61:195-200. 